\title{
Riego complementario y su efecto en la floración y producción de café (Coffea arabica L.) variedad CATRENIC, Carazo, Nicaragua, 2013-2014
}

\author{
Supplemental irrigation and its effect on flowering and production of coffee (Coffea \\ arabica L.) var. CATRENIC, Carazo, Nicaragua, 2013-2014
}

Henry Alberto Duarte-Canales, Rodolfo Munguía-Hernández, Víctor Aguilar-Bustamante, Gerardo Murillo-Malespín, Emilio Marrero-García.

Facultad de Agronomía, Universidad Nacional Agraria

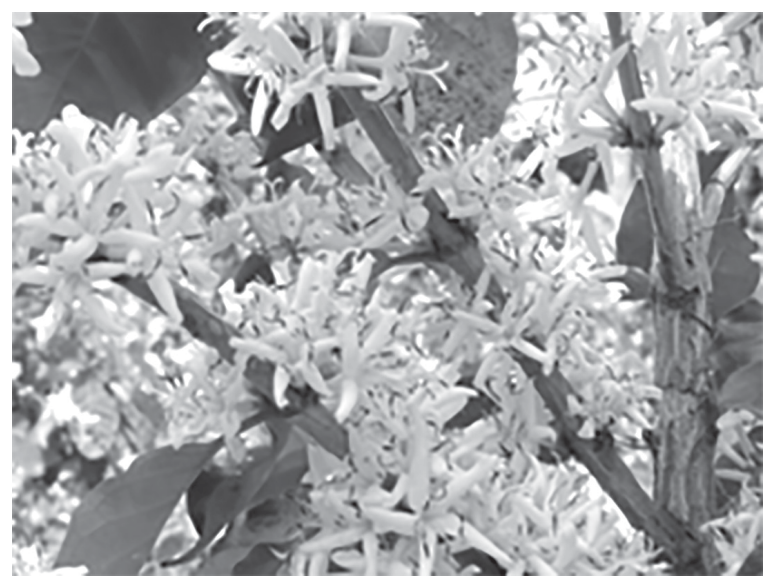

\section{RESUMEN}

Debido a la importancia que tiene el café (Coffea arabiga L.) en las exportaciones de Nicaragua, se estableció un experimento sobre

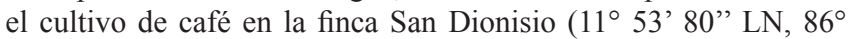
14' 05" LO y 552 msnm), San Marcos, Carazo, Nicaragua, con el propósito de evaluar el crecimiento, desarrollo y producción de café bajo tres láminas de riego por goteo complementario $(0,10,20 \mathrm{y}$ $30 \mathrm{~mm}$ ) aplicados a partir del 14 abril del 2013 hasta la presencia normal de las precipitaciones en la zona. Los tratamientos fueron ubicados en un Diseño Bloques Completos al Azar (BCA) con cuatro réplicas. Las variables desprendimiento de frutos, índice de área foliar, crecimiento de bandolas, formación de nudos potenciales y producción se aplicó un Análisis de Varianza (ANDEVA) y Tukey $(\alpha=0.05)$. La lámina de riego de $20 \mathrm{~mm}$ favoreció el crecimiento vegetativo, formación de estructuras productivas y productividad.

Palabras clave: Coffea arabica L., CATRENIC, láminas de riego.

\section{ABSTRACT}

Due to the importance of the coffee (Coffea arabica L.) in exports of Nicaragua, an experiment on the cultivation of coffee in the San Dionisio ( $11^{\circ} 53$ ' $80^{\prime}$ ' $\mathrm{LN}, 86^{\circ} 14^{\prime}$ ' $05^{\prime}$ 'was established LO and $552 \mathrm{~m}$ ), San Marcos, Carazo, Nicaragua, in order to evaluate the growth, development and production of coffee under three sheets of supplementary drip irrigation $(0,10,20$ and $30 \mathrm{~mm})$ applied from 14 April 2013 to the normal presence of rainfall in the area. The treatments were placed in a randomized complete block design (BCA) with four replicates. The variables fruit peel, leaf area index, bandolas growth, formation of knots and production potential analysis of variance (ANOVA) and Tukey ( $\alpha=$ 0.05 ) was applied. The irrigation depth of $20 \mathrm{~mm}$ favored vegetative growth, formation of productive structures and productivity Keywords: Coffea arabiga L., CATRENIC, water depths, BCA, ANOVA.

Recibido: 26 de mayo del 2016

Aceptado: 29 de septiembre del 2016 
$\mathrm{E}$ 1 café (Coffea arabica L.) es el rubro de mayor importancia en el sector agrícola de Nicaragua, ocupa el sexto lugar en el PIB nacional con un $18.2 \%$ de las exportaciones totales. Genera aproximadamente 300 mil empleos directos e indirectos que representan el 53 $\%$ del total de empleos del sector agropecuario y $14 \%$ del total de empleos a nivel nacional. El $93 \%$ son pequeños productores ( 0.7 a 3.5 ha) de 43 mil productores de café, ubicados principalmente en los departamentos de Jinotega, Matagalpa, Las Segovias y parte del Pacífico Sur. El rendimiento medio anual de café es de 646-711 kg ha-1 (MAGFOR, 2013).

Según FAO (2008), el cambio climático es una alteración del clima atribuido directa o indirectamente a la actividad humana. Dicho fenómeno conlleva una tendencia generalizada en la reducción de la producción agropecuaria, ocasionando pérdidas en los últimos años en los países de Centroamérica (FAO, 2008). Esto amenaza la seguridad alimentaria de los países, por lo que se tienen que desarrollar estrategias de adaptación del cultivo del café a los efectos del cambio climático.

El uso del riego por goteo complementario (Silva, 2006), incrementa la producción, reduce el aborto floral y caída del fruto hasta en un $57 \%$; hay ahorro de agua, energía, mano de obra, automatización de sistema y posibilidad de adaptar a las condiciones del suelo y topografía, así como el empleo de fertirrigación, lo que haría sostenible al sistema de cultivo del café.

Considerando lo anterior, se estableció el presente estudio con el objetivo de evaluar tres láminas de riego por goteo complementario sobre el estímulo de la floración y producción de café en la variedad CATRENIC, Finca San Dionisio, San Marcos, Carazo 2013.

\section{MATERIALES Y MÉTODOS}

Se estableció en la zona cafetalera del Pacifico Sur de Nicaragua, específicamente en la finca San Dionisio, San Marcos, Carazo, en coordenadas $11^{\circ} 53^{\prime} 80^{\prime \prime}$ latitud Norte y $86^{\circ}$ $14^{\prime} 05^{\prime}$ " longitud Oeste y $525 \mathrm{msnm}$. El periodo lluvioso en el año 2013 tuvo un acumulado de 1,301.75 mm; siendo el mes de septiembre el más húmedo con $343.41 \mathrm{~mm}$. Las temperaturas oscilaron entre los 20 y $28^{\circ} \mathrm{C}$.

El diseño utilizado fue en Bloques Completo al Azar (BCA) y cuatro réplicas. Los tratamientos distribuidos al Azar fueron: Testigo, 10, 20 y $30 \mathrm{~mm}$ de agua aplicados a partir del 14 de abril del 2013, hasta el establecimiento normal de las precipitaciones.

Asimismo, se consideró un diagnóstico de las condiciones hidrofísicas del suelo con los indicadores: infiltración del suelo determinado por medio del doble cilindro, capacidad de campo a través del método de la olla de Richard; así como la densidad real y aparente del suelo y porosidad.

La biometría obtenida en el cultivo fueron: índice de área foliar $\left(\mathrm{m}^{2}\right)$ con mediciones de 10 plantas y aplicado un factor de 0.7243 . La longitud de bandola (cm) obtenida en cinco plantas por tratamiento en la parte alta, parte media y parte baja. Se contabilizaron los nudos potenciales, nudos potencialmente productivos y nudos productivos del año anterior en las bandolas.

Antes de la ocurrencia de la apertura floral, fue contabilizado el número de yemas florales en seis bandolas de cinco plantas. Durante la floración ocurrida, 8 a 10 días después de la aplicación del primer riego, se identificaron las flores abiertas de las bandolas. Cada 30 días fueron cosechados los frutos por bandolas hasta la maduración ocurrida durante el período noviembre 2013 enero 2014. Toda la presente información recolectada permitió obtener la magnitud del derrame de frutos.

El corte de los frutos maduros y pesados en grano uva (1 libra), se realizó en 18 plantas por tratamientos correspondientes a los dos surcos centrales. En esta misma muestra fueron separados los granos dañados por insectos, enfermos $\mathrm{y}$ afectaciones en el fruto por las condiciones de clima para cada uno de los tratamientos. Se consideran los frutos dañados, los "chasparreados" (quemados por el sol), afectados por broca y frutos vanos (granos verdes y maduros).

Una muestra de $2 \mathrm{~kg}$ de grano uva por cada tratamiento, fue despulpada, fermentada, lavada y secada a temperatura ambiente durante dos meses hasta alcanzar el $12 \%$ de humedad y después determinado el rendimiento en $\mathrm{kg} \mathrm{ha}^{-1}$ de grano pergamino seco. Posterior a esto, se aplicó la metodología propuesta por ANACAFE (2011), para determinar el rendimiento en $\mathrm{kg} \mathrm{ha}^{-1}$ grano oro.

La información biométrica fue sometida a un análisis de ANDEVA y pruebas de rangos múltiples de Tukey $(\infty=0.05)$ utilizando el software Statistical Análisis System (SAS, v. 9.1).

\section{RESULTADOS Y DISCUSIÓN}

Velocidad de infiltración. La infiltración básica del suelo fue de $28 \mathrm{~mm} \mathrm{~h}^{-1}$ y la velocidad de infiltración es $42.6 \mathrm{~mm}$ $\mathrm{h}^{-1}$, esto quiere decir que es un suelo con un buen drenaje que puede soportar una intensidad de lluvia de $42.6 \mathrm{~mm}$ en una hora para que pueda saturarse. Esto se debió a que la clase textural del suelo es $\mathrm{n}$ franco arenoso, pero con baja retención de humedad (CENTA, 2013). 


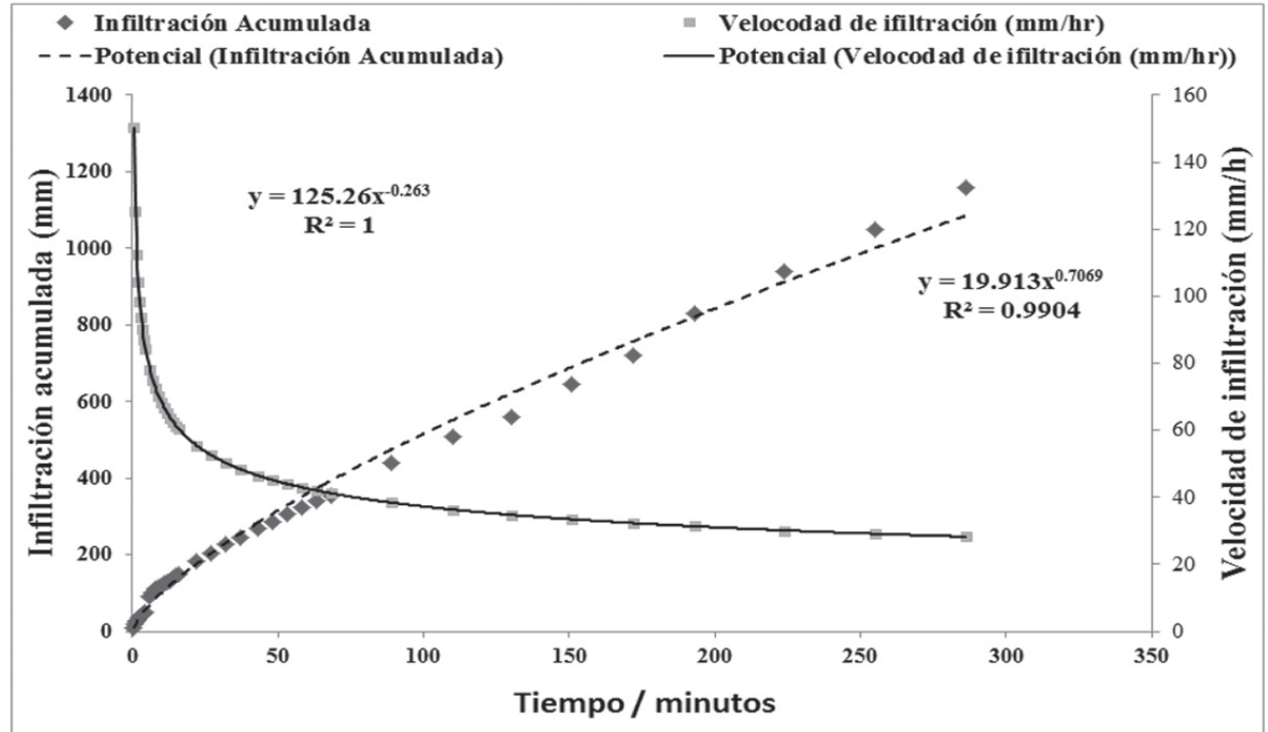

Figura 1. Curva de velocidad de infiltración e infiltración acumulada, suelo finca San Dionisio, Carazo, 2013.

La capacidad de retención de agua del suelo fue baja con $20.33 \%$. Blair (2006), afirma que los suelos francos arenosos retienen humedad entre 7.5 y $20.5 \%$. La densidad aparente y real fue de $1.35 \mathrm{~g} / \mathrm{cm}^{3}$ y $2.53 \mathrm{~g} / \mathrm{cm}^{3}$, respectivamente. Carpo y Fundora (1994), afirman que estos parámetros están influenciados por la densidad de las partículas del suelo mineral, cantidad de materia orgánica, compactación del suelo, actividades biológicas y abundancia de raíces. La porosidad de $47.26 \%$, lo que indica que el suelo tiene una buena circulación de agua y aire.

Índice de área foliar del dosel. La primera medición del área foliar del café mostró que la aplicación de agua por goteo con lámina de $30 \mathrm{~mm}$ superó en un $60.5 \%$ al testigo. Las láminas de 10 y $20 \mathrm{~mm}$ presentaron los menores valores promedios. En el segundo momento de medición se realizó en octubre del 2013, el área foliar fue afectada por las láminas de 20 y $30 \mathrm{~mm}$ con área foliar de $6.53 \mathrm{a} 6.56 \mathrm{~m}^{2}$, respectivamente (cuadro 1).

Cuadro 1. Efectos principales para la variable área foliar del cultivo de café en dos momentos de evaluación finca San Dionisio, Carazo, 2013

\begin{tabular}{|c|c|c|c|c|c|}
\hline & \multirow[t]{2}{*}{ Láminas de agua } & \multicolumn{4}{|c|}{ Área Foliar $\left(\mathrm{m}^{2}\right)$} \\
\hline & & $14-05-13$ & $\%$ & $11-10-13$ & $\%$ \\
\hline \multirow{5}{*}{ Riego } & Testigo & $1.24 \mathrm{~b}$ & 0.00 & $4.06 \mathrm{~b}$ & 0.00 \\
\hline & $10 \mathrm{~mm}$ & $1.40 \mathrm{ab}$ & 13.00 & $4.65 \mathrm{~b}$ & 15.00 \\
\hline & $20 \mathrm{~mm}$ & $1.52 \mathrm{ab}$ & 22.58 & $6.53 \mathrm{a}$ & 60.84 \\
\hline & $30 \mathrm{~mm}$ & $1.99 \mathrm{a}$ & 60.48 & $6.56 \mathrm{a}$ & 61.58 \\
\hline & $P r>F$ & 0.025 & & 0.032 & \\
\hline
\end{tabular}

Nudos y crecimiento de ban-

dola. El muestreo se realizó cuando el cultivo tenía formado la estructura productiva y por esta razón no se encontró diferencias estadísticas en ninguno de los tratamientos evaluados, debido a que todas las plantas estaban en las mismas condiciones de humedad del suelo, los promedios de nudos productivos oscilaron entre 11.47 y 12.93 nudos (cuadro 2).

Los nudos productivos varían cada año hacia el extremo de las bandolas (crecimiento plagiotrópico) y en sentido vertical del tallo (crecimiento ortotrópico), acumulando números de nudos improductivos y defoliados de la cosecha anterior (Ramírez, 1996).

Tanto las variables de crecimiento de bandolas y nudos potenciales son la respuesta a la aplicación de agua a través del manejo del riego, el cual inició el 15 de abril del 2013. Las variables número de nudos potencialmente productivos y crecimiento de bandola, fueron cuantificadas el 11 de octubre del 2013.

El número promedio de nudos potencialmente productivos en el testigo fue inferior estadísticamente con respecto a los tratamientos con riego. Los tratamientos con riego por goteo se obtuvieron resultados similares, ubicándose dentro de una misma categoría estadística. Los promedios de nudos potencialmente productivos más altos correspondieron a las láminas de 30 y 10 mm con 6.03 y 5.92, respectivamente.

El crecimiento de las bandolas estuvo directamente relacionado con el indicador anterior, a mayor crecimiento plagiotrópico de las bandolas, mayor fue el número de nudos potencialmente productivos formados. En el cuadro 2, los mayores valores fueron de $20.03,19.78$ y 18.67 $\mathrm{cm}$ correspondiente a las láminas de 10, 30 y $20 \mathrm{~mm}$, respectivamente. Estos resultados coinciden con los Cisneros y Martínez (2001). 
Cuadro 2. Efectos principales para la variable nudos productivos, nudos potenciales y crecimiento de bandola en el cultivo de café finca San Dionisio, Carazo, 2013

\begin{tabular}{ccccc}
\hline Láminas de agua & $\begin{array}{c}\text { Nudos } \\
\text { Productivos }\end{array}$ & $\begin{array}{c}\text { Nudos } \\
\text { Potenciales }\end{array}$ & $\begin{array}{c}\text { Crecimiento } \\
\text { Bandolas (cm) }\end{array}$ \\
Riego & Testigo & $11.48 \mathrm{a}$ & $3.63 \mathrm{~b}$ & $13.18 \mathrm{~b}$ \\
& $10 \mathrm{~mm}$ & $11.50 \mathrm{a}$ & $5.92 \mathrm{a}$ & $20.03 \mathrm{a}$ \\
& $12.93 \mathrm{a}$ & $5.66 \mathrm{a}$ & $18.67 \mathrm{a}$ \\
& $20 \mathrm{~mm}$ & $11.47 \mathrm{a}$ & $6.03 \mathrm{a}$ & $19.78 \mathrm{a}$ \\
\hline & 0.064 & 0.042 & 0.047 \\
\hline
\end{tabular}

Derrame productivo del café en riego por goteo. La aplicación de las láminas de riego se inició el 15 de abril del 2013, y la floración comenzó a partir de los 9 días después de que se aplicó riego, el testigo inició el proceso de floración 10 días después de haberse iniciado las primeras lluvias (12 de mayo del 2013).

La figura 2, muestra que durante los 24 días después de la floración (ddf) ocurrió con una mayor intensidad el derrame de los frutos. Las menores pérdidas de frutos ocurrieron en la lámina de $30 \mathrm{~mm}$ (36\%), lámina de $20 \mathrm{~mm} \mathrm{(46 \% )}$ y la lámina de $10 \mathrm{~mm}(66 \%)$. El testigo tuvo una pérdida de $24 \%$ de frutos totales, resultado que se debió a normalización de las precipitaciones ya la floración ocurrió cuando se dieron las primeras lluvias.

A los 38 días continúo el derrame de frutos, pero en este caso los tratamientos con riego lo hicieron con menor intensidad. El tratamiento con la lámina de $30 \mathrm{~mm}$ perdió el $0.95 \%, 20 \mathrm{~mm} 7 \%, 10 \mathrm{~mm} 1.13 \%$, y el testigo perdió hasta un $30 \%$. Según CENICAFE (2010), dos factores que afectan el desarrollo de la flor son el déficit hídrico durante la iniciación de la floración y la alta radiación solar, lo que está estrechamente relacionado con la capacidad de retención de agua del suelo y velocidad de infiltración; por otro lado, las plantas de café del ensayo a pleno sol con porcentaje de sombra bajo $(20 \%)$ permitió que hubiera mayor impacto de la radiación solar.

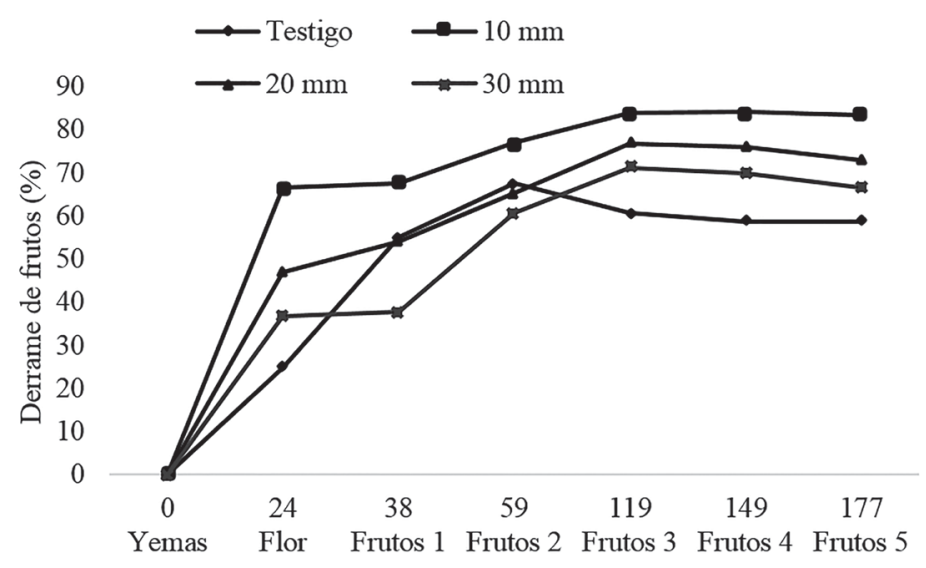

Figura 2. Derrame productivo en el cultivo de café, finca San Dionisio, San Marcos, Carazo, 2013.
Calidad física del grano. Los parámetros que se cuantificaron para conocer la calidad física del grano de café, no mostraron diferencias estadísticas. Los mejores tratamientos se obtuvieron en la lámina de 10 mm con un total de 343 frutos/ libra, similar a la lámina de 30 mm con 342.25 frutos/libra.

La cantidad de frutos verdes fue menor en los tratamientos de 20 y $10 \mathrm{~mm}$ con 0.38 y $0.63 \%$, respectivamente. UNICAFE (1995), menciona que los frutos verdes se caracterizan por tener menor peso, esto conlleva a menos tonelajes cosechados, adicionalmente es un indicador de la calidad del corte y recolección de los frutos. Para el caso del porcentaje de frutos pintos (madurez fisiológica) los promedios más altos se obtuvieron las láminas de 20 y $30 \mathrm{~mm}$ con 4.44 y 4.13 $\%$, respectivamente.

Un indicador importante dentro de los parámetros de calidad es el porcentaje de frutos maduros. En este estudio el rango de maduración fue entre 93.16 y $96.17 \%$, el valor más alto correspondió a la lámina de $20 \mathrm{~mm}$.

El tratamiento que obtuvo mejores resultados al cuantificar el porcentaje de frutos secos fue el testigo con un $0.67 \%$ de frutos secos, caso contrario con la lámina de $30 \mathrm{~mm}$ que obtuvo un resultado menos deseable con 4.76 \%. Según UNICAFE (1996), la presencia de frutos secos es consecuencia de daños ocasionados por la radiación solar o porque la cosecha no se hace en el tiempo requerido de corte y el grano se seca en la planta. La lámina de riego de $20 \mathrm{~mm}$ obtuvo un $28.58 \%$ de frutos afectados por brocas, la lámina de $10 \mathrm{~mm}$ produjo un promedio alto con $23.53 \%$, y la lámina de $30 \mathrm{~mm}$ con un $10.46 \%$ fue la que presentó el promedio más bajo. Alvarado y Roja (1998), mencionan que cuando el fruto es atacado por broca en estado verde y maduro no cae pero pierde peso, se convierte en café vano, de baja calidad y produce una apreciable disminución en el rendimiento.

En el cuadro 3 se expresan los porcentaje de frutos flotantes en los diferentes tratamientos fue muy similar, el valor más alto fue de $13.89 \%$ correspondiente al testigo y el valor más bajo fue de $10.42 \%$ perteneciente a la lámina de $10 \mathrm{~mm}$. Para el caso del porcentaje de frutos manchados el valor menor deseable lo obtuvo la lámina de $10 \mathrm{~mm}$ con $7.72 \%$ y el promedio más bajo correspondió a la lámina de $20 \mathrm{~mm}$ con $2.15 \%$. 
Cuadro 3. Efectos principales para la variable calidad física del grano en el cultivo de café bajo riego por goteo finca San Dionisio, Carazo, 2013

\begin{tabular}{lrrrrr}
\hline \multicolumn{1}{c}{ Indicador } & Pr $>\mathrm{F}$ & \multicolumn{4}{c}{ Tratamientos } \\
\cline { 2 - 6 } & & Testigo & $10 \mathrm{~mm}$ & $20 \mathrm{~mm}$ & $30 \mathrm{~mm}$ \\
\hline Frutos por libra & 0.21 & 335.25 & 343.00 & 330.00 & 342.25 \\
\%Frutos Verdes & 0.88 & 1.15 & 0.63 & 0.38 & 0.74 \\
\%Frutos Pintos & 0.19 & 5.67 & 5.55 & 3.44 & 4.13 \\
\%Frutos Maduros & 0.25 & 93.16 & 93.81 & 96.17 & 95.12 \\
\%Frutos Secos & 0.07 & 0.67 & 1.00 & 2.17 & 4.76 \\
\%Frutos Brocados & 0.14 & 14.11 & 23.53 & 29.58 & 10.46 \\
\%Frutos Flotantes & 0.06 & 13.89 & 10.42 & 12.95 & 12.56 \\
\hline \%Frutos Manchados & 0.56 & 3.56 & 7.72 & 2.15 & 3.76 \\
\hline
\end{tabular}

Rendimiento del grano. Los rendimientos obtenidos con un mayor valor correspondieron a las láminas de riego de 20 $\mathrm{mm}, 30 \mathrm{~mm}, 0 \mathrm{~mm}$ y el riego de $10 \mathrm{~mm}$ fue menor; sin embargo, no hubieron diferencias estadísticas entre sí (cuadro 4). Es importante mencionar que los rendimientos obtenidos por este último tratamiento presentaron los mayores porcentajes de derrame productivo (figura 2). La relación uva pergamino le corresponde al testigo seguido por los tratamientos 30,20 y $10 \mathrm{~mm}$.
ANACAFE (2011), afirma que la cascarilla suelta o pergamino representa el $5 \%$ del peso total del grano de café. Según Oliveira (2007), se obtiene mayor producción de grano entre mayor sea la cantidad de agua aplicada. Silva (2006), este obtuvo menor rendimiento que el testigo, el cual obtuvo los mejores rendimientos en un plazo de cuatro años. Bonomo et al., (2008), confirmaron que el empleo de riego proporciona aumentos en la productividad media de café.

\section{CONCLUSIONES}

Las propiedades hidrofísicas del suelo de la finca "San Dionisio" es de una clase textural franco arenoso, con una capacidad de campo de $20.3 \%$ y una infiltración básica de $28 \mathrm{~mm} / \mathrm{h}$ siendo un suelo muy permeable lo que se traduce en una velocidad de infiltración alta $(42.6 \mathrm{~mm} / \mathrm{h})$ y una baja capacidad de retención de agua. El riego por goteo en café superó hasta en un $60.48 \%$ al testigo, en crecimiento vegetativo. Los mayores rendimientos se obtuvieron en la lámina de 20 y $30 \mathrm{~mm}$ con un $20 \%$ y $8 \%$ en relación al testigo.

Cuadro 4. Rendimiento en $\mathrm{kg} \mathrm{ha}^{-1}$ y relación uva pergamino de los tratamientos evaluados finca San Dionisio, Carazo, 2013

\begin{tabular}{cccc}
\hline \multirow{2}{*}{ Tratamiento } & \multicolumn{2}{c}{ Rendimiento en kg ha ${ }^{-1}$} & \multirow{2}{*}{ Relación uva pergamino } \\
\cline { 2 - 3 } & Grano uva & Grano Pergamino & \\
\hline Testigo & 6878.59 & 1255.07 & 0.1875 \\
$10 \mathrm{~mm}$ & 6168.81 & 1107.07 & 0.1775 \\
$20 \mathrm{~mm}$ & 8176.02 & 1503.93 & 0.1850 \\
$30 \mathrm{~mm}$ & 7337.43 & 1352.66 & 0.1850 \\
\hline$p>\mathrm{F}$ & 0.067 & & \\
\hline
\end{tabular}

\section{REFERENCIAS BIBLIOGRÁFICAS}

ANACAFE (Asociación nacional de cafetales, NI). 2011. Los subproductos del café. (en línea). Consultado 18 jul. 2014. Disponible en www.anacafe.org/glifos/index.php/beneficiohumedo subproductos

Alvarado, M; Rojas, G. 1998. El cultivo y beneficiado de café. 2 ed. Ed. Universidad San José, CR. 160 p.

Blair, N. 2006. Long-term management impactions on soil C, N and physical fertility. Part I: broadbalk experiment. Soil Till Res 91 : 30-38.

Bonomo, R; Oliveira, C; Silveira, N; Bonomo, P. 2008. La productividad de café Arábica de regadío en el Cerrado de Goiás. Agronómico Tropical de Investigación, v.38.

Carpo, P; Fundora H, O. (1994). Edafología. Habana, CU. 300p.

CENICAFE (Centro Nacional de Investigaciones de Café). 2010. Anuario meteorológico cafetero. p 200-320.

CENTA (Centro Nacional de Tecnología Agropecuaria y Forestal) 2013. Riego por goteo. San Salvador, Salv. 98p.

Cisneros, E; Martínez, R. 2001. Respuesta del cafeto al riego por goteo en plantaciones de fomento. Revista Ciencias Técnicas Agropecuarias, Vol 10. Universidad Agraria de La Habana, Cuba.

Ramírez, JE. 1996. Estudios de sistemas de podas de café oor hileras y por lotes. Agronomía costarricense 20(2):167-172.

UNICAFE (Unión Nicaragüense de Cafetaleros). 1995. Principales defectos del grano que influyen en la calidad del café. In: El Caficultor. Año $3 \mathrm{~N}^{\circ}$ 10. Vicegerencia de Comercialización (Laboratorio de Catacion) Managua, Nicaragua. p 6.

UNICAFE (Unión Nicaragüense de Cafetaleros). 1996. Manual de Caficultura Nicaragüense. Primera Edición. Managua, Nicaragua. Editorial CENACOR. 243 p. 
Silva, A; Faria, M. 2006. Viabilidade técnico-economica do uso do sistema de irrigacão por gojetamiento na cultura do cafeeiro. Revista brasileira de engenharia agrícola e ambiental, campina, v.7. n1

MAGFOR (Ministerio Agropecuario y Forestal). 2013. El café en Nicaragua. Programa Nacional de Fomento a la Producción Sostenible de Café Arábigo. Consultado 28 de marzo 2014. Disponible en:http://www.google.com.ni/url?sa=t\&rct=j\&q=\&esr$\mathrm{c}=\mathrm{s} \&$ source=web\&cd=1\&ved=0CBsQFjAA\&url=http $\% 3 \mathrm{~A} \% 2 \mathrm{~F} \% 2 \mathrm{Fwww}$.magfor.gob.ni\%2Fdescargas $\% 2 \mathrm{Fpublicaciones} \% 2 \mathrm{~F}-$ cafecacao\%2Fcafenicaragua.pdf\&ei=oi0sVMH0GdKqggT2ILABw\&usg=AFQjCNHHIXLe3dokTJWGxbhFAC8N9Ad4aQ\&bvm=bv.76477589,d.eXY.

Oliveira, E; Faria, M. 2007. Manejo e viabilidade econômica da irrigação por gotejamento na cultura do cafeeiro acaiá considerando seis safras. Goias, Br. 